\title{
Persistence of sham feeding after intragastric meals in the rat: Concentration-intake relations
}

\author{
DOUGLAS G. MOOK, AMY OBERLIN, ERIN TYNDALL, and SUE WAGNER \\ University of Virginia, Charlottesville, Virginia
}

\begin{abstract}
In rats sham fed through esophageal fistulas, responsiveness to sucrose over a range of concentrations was assessed under two conditions: immediately following a large intragastric (IG) preload of liquid diet, and after $10 \mathrm{~h}$ of deprivation with no preload. The preload had no reliable effect on sucrose sham feeding at any sucrose concentration. Therefore, the insensitivity of sham feeding to IG loads is not confined to intense or highly palatable oral stimuli, but extends even to minimally effective ones.
\end{abstract}

In previous reports, we have described a series of experiments dealing with the effects of intragastric (IG) nutrient preloads on sham feeding in rats with esophageal fistulas. What we find is that sham feeding is quite insensitive to such preloads. First, the effect of an IG preload is all-or-none: If such a load fails to suppress sham feeding entirely, it does not affect it at all. Second, the all-ornone threshold is very high, equaling or exceeding spontaneous real-meal size. This is so even in rats maintained at normal body weight (Mook, Atkinson, Johnston, \& Wagner, in press). And it remains true even if the "behavioral context" (Blass \& Hall, 1976) of the IG preload is restored-that is, if the preload is "real fed" rather than received passively (Mook, Yoo, \& Wagner, in press).

In all of these studies, the material offered for ingestion was intense and palatable as a gustatory stimulus: $1 \mathrm{M}$ glucose, or a milk-based liquid diet. It is conceivable that ceiling effects obscured a suppressant effect of the IG load that would be visible if less palatable material were offered. Or, ceiling effects aside, the insensitivity to gastric loads may apply only to highly palatable commodities.

We now show that this is not so. In the present experiments, we offered sucrose at varying concentrations for sham ingestion, immediately following a large IG preload of liquid diet. In comparison with a no-preload condition, the IG preload had little or no effect on sham feeding of sucrose at any concentration, even one that was minimally effective as a stimulus for ingestion.

\section{GENERAL METHOD}

\section{Subjects}

Seven female Sprague-Dawley rats, with a preoperative weight of $265-320 \mathrm{~g}$, served as subjects. They had received the second stage of surgery (see below) at least 1 week before the present experiments began. During the intervening time, they learned to sham

Correspondence should be addressed to D. Mook, Department of Psychology, 102 Gilmer Hall, University of Virginia, Charlottesville, VA 22903-2477. feed (Davis \& Smith, 1990) with $1 \mathrm{M}$ glucose as the stimulus material. Throughout the present experiments, they were maintained at about $85 \%$ of their preoperative weights (see below).

\section{Surgery}

The esophagostomy preparation has been described in detail elsewhere (Mook \& Wagner, 1991) and will be described only briefly here. The procedure is done in two stages. In the first, a Silastic cannula ( 0.065 o.d.) is implanted into the stomach, held there by a purse-string suture and a $1-\mathrm{cm}$ square of Mersilene mesh, caulked to the tubing and sutured to the stomach wall. The cannula is exteriorized between the scapulae; there it is held in place by a rectangle of Marlex mesh, caulked to the tubing and implanted under the skin.

In the second stage, the esophagus is transected through a midline incision in the throat. The lower end is closed with a suture ligature, and the upper end is sutured to an oval paramidline wound to provide a natural fistula. All surgery is performed under methoxyflurane anesthesia.

\section{Apparatus and Maintenance}

The rats were tested in their home cages. These were of wire mesh, $20 \times 25 \times 18 \mathrm{~cm}$ high, with sliding mesh tops. A tether, consisting of PE 90 with connectors and protective spring wire, was connected to each rat's cannula and protruded through the top of the cage, where it was counterweighted and connected in turn by flexible tubing to the peristaltic pump that delivered maintenance rations of liquid diet. The tether was interrupted by a water-tight swivel joint. The swivel and the light, flexible tether permitted the rat complete freedom of movement within its cage. This arrangement is described in detail elsewhere; so is the maintenance diet, a milk-based fluid that supplies about $1.1 \mathrm{kcal} / \mathrm{ml}$ at full strength (Mook \& Wagner, 1991).

Esophagostomized rats depend on IG infusions for nutritional and hydrational maintenance. In all the experiments reported here, the rats were maintained with five liquid-diet rations or "meals," delivered IG by the pump, which in turn was controlled by automatic timing devices. Each meal was $20 \mathrm{ml}$ in volume, delivered at $1.1 \mathrm{ml} / \mathrm{min}$. The meals were delivered at 3 -h intervals from onset to onset, beginning at noon; the last of the five meals thus occurred at midnight. The rats were then deprived of systemic food and water until noon the following day, when the cycle began again.

A total of about $60 \mathrm{ml}$ of diet was delivered each day in a total fluid volume of $100 \mathrm{ml}$, but diet concentration was adjusted slightly each day as was necessary, to maintain constancy of body weight. 
In addition, for the present experiments we modified the regimen as follows: For the first two meals each day, the diet was diluted by only $10 \%$ with water; these meals consisted of $90 \%$ diet. After those two meals, the remaining diet ration was further diluted with water as was required. Thus, meals after the first two were more dilute - and to a variable extent-than the first two. We did this because we wished to see the effect of the first two meals on sham feeding (see below); and, by increasing their caloric density, we reasoned that we should make their effect more readily visible if it were there to be seen.

Despite the 10-h deprivation, rats maintained on this regimen are behaviorally unresponsive to water, as can be shown by occasional spot checks in which water is offered for sham ingestion and is invariably refused. This is true after an IG meal as well as before one. That is why we speak of the ingestive response to sweet solutions as sham feeding rather than sham drinking.

\section{Procedure}

Sham feeding. For sham-feeding tests, a graduated cylinder with a metal drinking spout was clipped to the front of the animal's cage. Fluid lapped from the cylinder escaped through the esophageal fistula and did not enter the stomach. Sham intake was read to $1 \mathrm{ml}$, at 10-min intervals for $40 \mathrm{~min}$.

"Real feeding." As preparation for Experiment 2 below, a session was conducted in which the conditions of real feeding were simulated. This was done by connecting the inlet to the rat's tether (which in turn led to the IG cannula) through flexible tubing to a hand-held syringe. The syringe and tubing were filled with the fluid to be infused. As the rat lapped from the drinking cylinder, losing what it ingested through the fistula, equivalent volumes of fluid were delivered into its stomach from the syringe. Continuous monitoring of the cylinder and syringe calibrations permitted a continuous match between the amount fed by mouth and the amount infused IG (a much closer match is possible in this way than with an automated pumping system; see Mook, 1963).

Preload and control conditions. Recall that the rats were fed IG "'meals," by an automatic pumping system, beginning at noon and ending at midnight. For the present experiments, we treated the second meal of the day (the 3 p.m. one) as an IG preload, asking, What effect has that meal on sham intake of a sucrose solution, in comparison with a no-preload control condition?

On preload days, therefore, the sucrose solution was offered immediately following the 3 p.m. meal. Thus the preload consisted of a large IG meal, presented at a physiological interval after a prior meal. On control days, the sucrose solution was offered at $10 \mathrm{a} . \mathrm{m}$. instead, after $10 \mathrm{~h}$ of deprivation and before that day's maintenance meals began.

Technically, this procedure does confound experimental condition (preload vs. none) with time of day ( 3 p.m. vs. 10 a.m.). The alternative would be to skip two meals on each control day, and this we were reluctant to do. The consistency of the data, here and in our other experiments with this method (Mook et al., in press; Mook, Wagner, \& Hartline, 1991) suggests that the confound is not serious.

Further procedural details are given for the individual experiments.

\section{EXPERIMENT 1}

In this first experiment, we examined the effects of an IG diet preload on the sham-feeding response to sucrose over a range of concentrations.

\section{Method}

The experiment was conducted over two 4-day blocks. Within each block, sucrose was offered for sham ingestion at 1.0, 0.5, 0.25, and $0.125 \mathrm{M}$, in that order on successive days. For one 4-day block, sham feeding was tested in the morning after $10 \mathrm{~h}$ of deprivation (the control condition); for the other 4-day block, sham feeding was tested after the 3 p.m. meal (the experimental condition; see General Method). The order of blocks was counterbalanced across rats.

\section{Results}

Figure 1 shows concentration-intake functions under the two conditions. Total sham intake rose markedly with increasing sucrose concentration offered for sham ingestion. The effect of concentration was significant $[F(3,18)=41.04]$.

As for the experimental conditions, sham intake was slightly lower in the preload condition at all but the lowest sucrose concentration. However, the difference was not significant $[F(1,6)=4.25$, n.s. $]$, nor was there an interaction $(F=1.10$, n.s. $)$.

Intake at the lowest sucrose concentration $(0.125 \mathrm{M})$ was modest in volume and clearly below any "ceiling" on ingestion; yet the IG meal did not depress it any further. Nor was this a "floor" effect, for intake was significantly higher than zero under both conditions $[t(6) \geq 2.14, p<$ $.05]$. Therefore, sham intake could have been driven still lower by the preload meal. But it was not. What difference there was, was very small and in the wrong direction.

\section{Discussion}

In comparison with a no-preload control, sham intake of sucrose was not reliably affected by a large IG meal, presented at a normal interval following another such meal. This has been our consistent finding with strong

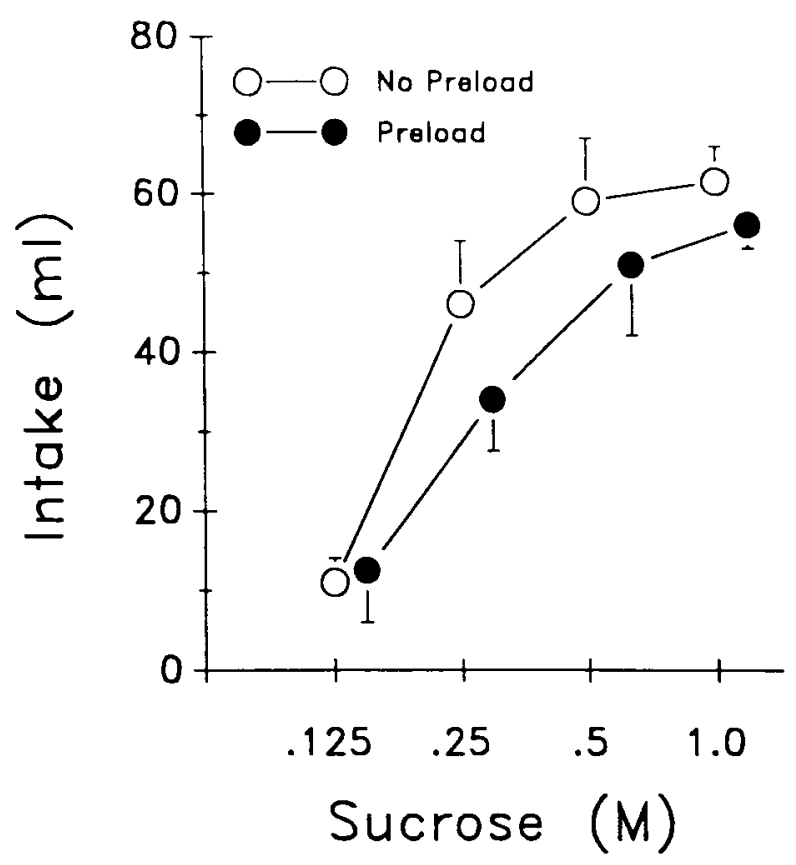

Figure 1. Sham intake as a function of sucrose concentration, after a 20-ml meal (preload) or with no prior meal (no preload). For clarity, the preload data are displaced to the right. 
carbohydrate solutions (Mook et al., in press). Now we see that it applies to weak ones as well.

\section{EXPERIMENT 2}

The IG meal, delivered as a "preload" in Experiment 1 , was roughly equal to spontaneous meal size of that diet in other rats comparably maintained (Mook et al., 1991). We did not yet know, however, how it compared with real-meal size in these particular rats. We therefore determined real-meal volumes in the present rats. Finding these somewhat larger than $20 \mathrm{ml}$, we then increased the size of the preloads to equal them, and repeated the experiment.

\section{Method}

One of the 7 rats died during the course of this experiment. All data are from the 6 rats remaining.

On the day in which real-meal size was measured, the noon meal was delivered IG as usual. At 3 p.m., however, rather than deliver another meal automatically, we permitted the rat to "real feed" its meal. Liquid diet (90\% of full strength) was offered in a drinking cylinder and, as the rat sham fed, we infused matching amounts of the same diet into the stomach (see General Method). This was done for 3 consecutive days.

All rats fed promptly and avidly when presented the liquid diet. And, as noted above, real-meal size was somewhat larger in these rats than has been our experience. It was stable over the 3 days, averaging $29.33,23.71$, and $27.0 \mathrm{ml}$ respectively. We therefore averaged, for each rat, the three meal volumes to obtain a single estimate of real-meal size. These estimates ranged from 24.6 to $30.3 \mathrm{ml}$, with a mean of $27.51 \mathrm{ml}$. Therefore, our $20-\mathrm{ml}$ preloads in Experiment 1 were, on the average, about $73 \%$ of estimated realmeal size.

Over the following 6 days, we then repeated the procedure of Experiment 1, with the following modifications: (1) the highest sucrose concentration (1 M) was omitted, since Experiment 1 yielded little difference between it and the next lower concentration. And (2) the 3 p.m. "preload" meal was increased in volume for each rat, to equal its real-meal size as previously estimated.

\section{Results}

As Figure 2 shows, once again sham intake was a steeply rising function of sucrose concentration, and again the effect was significant $[F(2,10)=52.42]$. As before, the effect of experimental condition was not significant $(F<1)$. The interaction was marginally significant, reflecting the "crossover" of the two functions $[F(2,10)=$ $4.75, p<.05]$. In light of the rest of the data (see Experiment 1 , and below), we judge this to be a Type I error.

Of particular interest is the response to the lowest sucrose concentration $(0.125 \mathrm{M})$, because, given the significant concentration effect, the amount sham fed here is again clearly below any "ceiling" on ingestion. Once again it is not at the "floor" either, for intake of that solution was significantly different from zero under both conditions $[t(5) \geq 2.62, p<.05]$.

In this experiment, as in the others reported here, the modest sham intake at $0.125 \mathrm{M}$ reflects primarily the early termination of the ingestive bout. At higher concentrations, intake proceeded at a roughly constant rate through- out the $40-\mathrm{min}$ test period. At $0.125 \mathrm{M}$, an initial rapid burst of feeding was replaced by cessation or, in some cases, episodic nibbling over the rest of the session. The rats took $85 \%$ (after preload) or $76 \%$ (without preload) of their total volume intake in the first $20 \mathrm{~min}$ of the session.

As to the effect of the IG preload at that concentration, inspection of the figure shows that, on the average, sham intake was lower after the preload by nearly $50 \%$. The effect was inconsistent, however; 2 of the 6 rats actually sham fed more under the preload condition. And it was nonsignificant even by post hoc $t$ test $[t(5)=1.50$, n.s. $]$.

\section{Replication 1}

In view of the ambiguous data at $0.125 \mathrm{M}$ (Figure 2), we replicated the experiment at that concentration only. On 2 consecutive days, the two experimental conditions were imposed in counterbalanced order, and sham intake of $0.125 \mathrm{M}$ was measured as before.

Sham intake after preload averaged $29.17 \mathrm{ml}$; it was $45.0 \mathrm{ml}$ under control conditions. As before, however, the difference was inconsistent in direction (reversed in 3 of the 6 rats) and nonsignificant $[t(5)=1.59$, n.s.].

\section{Replication 2}

Yet another replication was conducted over the following 3 days, in which the control condition (A) and the preload condition (B) were imposed in ABA order. This time, the effect was even smaller: sham intake averaged $26.67 \mathrm{ml}$ following the preload and $31.34 \mathrm{ml}$ without it $(t<1)$.

\section{Discussion}

An IG meal equal to spontaneous meal size had no effect on subsequent sham feeding of sucrose, except pos-

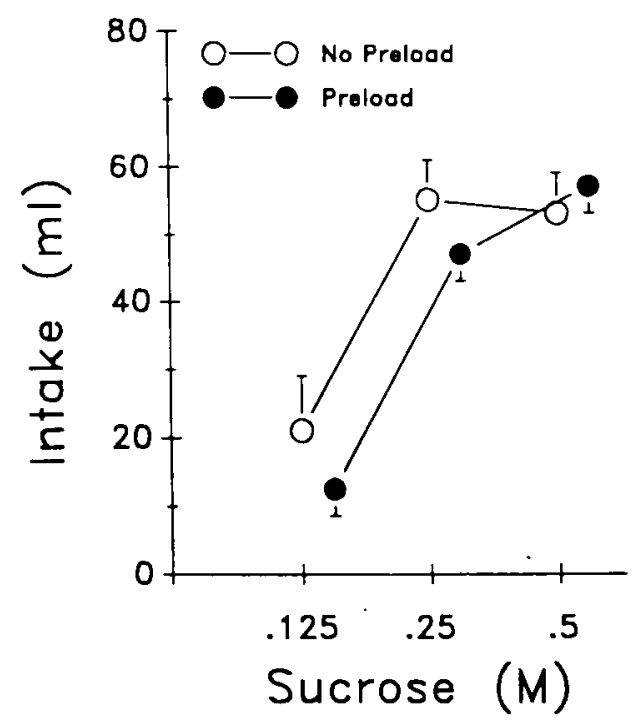

Figure 2. Sham intake as a function of sucrose concentration, anter a meal of normal size (preload) or with no prior meal (no preload). For clarity, the preload data are displaced to the right. 
sibly at the lowest concentration offered $(0.125 \mathrm{M})$. For that concentration, the average effect was in the direction of a suppressant effect of the meal, on each replication. However, also on each replication, the effect was variable in direction and statistically unreliable. If the IG meal does affect sham intake at that concentration, it certainly does not affect it consistently.

This is so, even though that solution evokes only a modest sham-feeding response even without the meal as preload. Certainly that response is far below any "ceiling" on sham intake.

More generally, it is perhaps not surprising that our IG loads failed to satiate the rats, even though the loads equaled normal meal size. It is well known that an IG load may be less than fully effective in producing satiety, whereas the same load taken by mouth would be (e.g., Kohn, 1951). "Less than fully effective," however, is one thing; "no effect at all" is another. If inhibition on ingestion is dose dependent (see below), we ought to have seen some consistent suppressant effect. No such effect was demonstrable.

\section{GENERAL DISCUSSION}

In all these experiments, we have seen that in our preparations, the sham-feeding response to sucrose simply was not reliably affected by the large IG loads of liquid diet immediately preceding. Sham intake was about the same whether the rat was 10-h food and water deprived or had had a full meal, approaching (Experiment 1) or equaling (Experiment 2) its spontaneous meal size, immediately prior to the sham-feeding test. Not only that, but in the latter case the preload meal followed another meal by an interval within the normal range. Yet no effect on sham feeding was demonstrable.

That this occurs at high concentrations offered for sham feeding replicates our previous findings (Mook et al., in press; Mook, Yoo, \& Wagner, in press). Now we have further shown that it happens even when minimally effective oral stimuli are presented to the mouth.

At high concentrations, sucrose evokes a copious and continuous sham-feeding bout that proceeds at a constant rate for $40 \mathrm{~min}$ or more. Our most dilute sucrose concentration $(0.125 \mathrm{M})$ supports only a modest sham-feeding bout, lasting $20 \mathrm{~min}$ or less and about $20-30 \mathrm{ml}$ in volume. Yet even a large IG preload does not seem to make the response any more modest than that. All this suggests that until a very high threshold is exceeded, the postingestive effects of an IG meal simply make no contact with the mechanisms by which oral stimuli, weak or strong, drive the ingestive response.

Such insensitivity to systemic stimuli poses a problem, for other investigators have found sham feeding to be quite sensitive to systemic manipulations such as intraperitoneal injections of glucose (Bedard \& Weingarten, 1989), intraduodenal infusions of food (Liebling, Eisner, Gibbs, \& Smith, 1975), or manipulations of neurotransmitter sys- tems (e.g., Geary \& Smith, 1985; Schneider, Davis, Watson, \& Smith, 1990). These affect sham feeding in a dosedependent way, which means that some effect is seen well before the maximum effect is.

Geary and Smith (1985), for example, showed that sucrose sham feeding was quite sensitive to the dopamine receptor antagonist pimozide. Response to sucrose was depressed in a dose-dependent way by pimozide; and the effect was also modulated by what was offered for ingestion, such that high sucrose concentrations were treated as if they were lower. Similar data were obtained for treatment with cholecystokinin (Waldbillig \& O'Callaghan, 1980), another suppressor to which sham feeding is sensitive (Gibbs, Young, \& Smith, 1973).

Now all such manipulations are intended to activate or mimic some of the "cascade" of events that constitute the postingestive effects of feeding. But if they do so, and if sham feeding is sensitive to the components of this cascade, then it ought to be at least as sensitive to the whole thing, as initiated by a meal delivered to the stomach. In our hands at least, it is not.

(It may at first seem surprising that there are few data from other laboratories on the effects of gastric loads. We suspect this is because in most labs sham feeding is done through an open gastric fistula. This makes the effect of a gastric preload quite difficult to study, and we consider this to be one of the advantages of esophagostomy.)

One possible way of resolving the paradox is as follows. Our esophagostomized rats receive extended practice at sham feeding. In addition, they are exclusive sham feeders: Unless we arrange otherwise, they never experience systemic consequences from their acts of ingestion. Conversely, their nutritional and hydrational maintenance rations are delivered IG by automatic apparatus, without the ingestive act. Thus we remove all contingency between ingestive behavior and systemic repletion.

This state of affairs would be expected to break up conditioned or anticipatory controls over the ingestive bout. It is very likely that such controls operate, not only in normal feeding (cf. Weingarten, 1990), but also in the early stages of a rat's sham-feeding career. Sham feeding typically becomes copious only by the third or fourth sham-feeding session (Davis \& Smith, 1990; Mook, Culberson, Gelbart, \& McDonald, 1983); and the interpolation of real-feeding bouts between these sessions can greatly retard the process (Davis \& Smith, 1990). All this suggests that rats must "learn to sham feed" copiously (Davis \& Smith, 1990); or, perhaps better, that they must unlearn the "nutritive expectancies" (Fedorchak \& Bolles, 1988) that limit sham feeding initially.

If all this is so, then many studies of sham feeding, done with relatively naive sham feeders, may have confounded conditioned with unconditioned controls. This is especially likely when (as with the self-maintaining gastric-fistula preparation) sham feeding is also intermittent, with bouts of real feeding and drinking interpolated between experimental sessions. In contrast, the study of experienced 
and exclusive sham feeders may permit us to see, for the first time, what the unconditioned components of the system are like.

Perhaps that is what we are seeing in this and other recent experiments. It may be that the unconditioned component of the controlling system operates all-or-none, and is insensitive to gastric loads below a high threshold. Conversely, conditioned controls may make feeding more sensitive to internal manipulations than it otherwise would be. For the case of cholecystokinin, this has been shown directly (Fedorchak \& Bolles, 1988). In light of the present results, perhaps the principle is more general than we have supposed.

\section{REFERENCES}

Bedard, M., \& Weingarten, H. P. (1989). Postabsorptive glucose decreases excitatory effects of taste on ingestion. American Journal of Physiology, 256, R1142-R1147.

BLASS, E. M., HALL, W. G. (1976). Drinking termination: Interactions among hydrational, orogastic, and behavioral controls in rats. Psychological Review, 83, 356-374.

DAvis, J. D., SMITH, G. P. (1990). Learning to sham feed: Behavioral adjustments to loss of physiological postingestional stimuli. American Journal of Physiology, 259, R1228-R1235.

FedorchaK, P. M., \& Bolles, R. C. (1988). Nutritive expectancies mediate cholecystokinin's suppression-of-intake effect. Behavioral Neuroscience, 102, 451-455.

Geary, N., Smith, G. P. (1985). Pimozide decreases the positive reinforcing effect of sham fed sucrose in the rat. Pharmacology, Biochemistry \& Behavior, 22, 787-790.

Gibzs, J., Young, R. C., \& Smith, G. P. (1973). Cholecystokinin elicits satiety in rats with open gastric fistulas. Nature, 245, 323-324.
KoHN, M. (1951). Satiation of hunger from food injected directly into the stomach versus food ingested by mouth. Journal of Comparative \& Physiological Psychology, 44, 412-422.

Liebling, D. S., Eisner, J. D., Gibas, J., \& Smith, G. P. (1975). Intestinal satiety in rats. Journal of Comparative \& Physiological Psychology, 89, 955-965.

Mook, D. G. (1963). Oral and postingestional determinants of the intake of various solutions in rats with esophageal fistulas. Joumal of Comparative \& Physiological Psychology. 56, 645-659.

Mook, D. G., Atkinson, B., Johnston, L., \& Wagner, S. (in press) Persistence of sham feeding after intragastric meals in rats. Appetite.

Mook, D. G., Culberson, R., Gelbart, R. J., McDonald, K (1983). Oropharyngeal control of ingestion in rats: Acquisition of sham-drinking patterns. Behavioral Neuroscience, 97, 574-584.

MoOK, D. G., \& W AGNER, S. (1991). Preparation and maintenance of chronic esophagostomized rats: An update. Physiology \& Behavior. 50, 1083-1085.

MoOk, D. G., Wagner, S., \& Hartline, D. F. (1991). All-or-none suppression of glucose sham feeding by an intragastric mixed meal in rats. Behavioral Neuroscience, 105, 712-720

Mook, D. G., Yoo, D., W W W Ner, S. (in press). Persistence of sham feeding after real-fed gastric loads in rats. Appetite.

Schneider, L. H., Davis, J. D., Watson, C. A., \& Smith, G. P. (1990). Similar effect of raclopride and reduced sucrose concentration on the microstructure of sucrose sham feeding. European Journal of Pharmacology, 186, 61-70.

Waldbillig, R. J., O'Callaghan, M. (1980). Hormones and hedonics cholecystokinin and taste: A possible behavioral mechanism of action. Physiology \& Behavior, 25, 25-30.

Weingarten, H. P. (1990). Learning, homeostasis, and the control of feeding behavior. In E. D. Capaldi \& T. L. Powley (Eds.), Taste, experience, and feeding (pp. 14-27). Washington, DC: American Psychological Association.

(Manuscript received November 3, 1992; revision accepted for publication December 11, 1992). 